\title{
Brjuno Numbers and the Symbolic Dynamics of the Complex Exponential
}

\author{
R. E. Lee DeVille * \\ Department of Mathematical Sciences \\ Rensselaer Polytechnic Institute. \\ E-mail: devilr@rpi.edu
}

\begin{abstract}
We show that the set of itineraries for the complex exponential family $\lambda e^{z}$ is closely related, through the continued fraction expansion, to the set of Brjuno numbers.
\end{abstract}

Key Words: complex dynamical systems, Brjuno numbers, continued fraction expansion

\section{INTRODUCTION}

In this paper, we note a connection between number theory and dynamical systems, in that we will show an object which is associated to a given family of analytic maps also has an interesting number theoretic property. It was shown in [5] that there is a set of irrational numbers which are associated with the complex exponential family $\lambda e^{z}$. We will show below that this set is closely related to the Brjuno numbers.

The Brjuno numbers are a set of irrational numbers defined in the following manner: Given an irrational $\alpha$, generate the continued fraction convergents to $\alpha$, denoted by $\frac{p_{n}}{q_{n}}$. The sequence $\left\{q_{n}\right\}$ is well-defined and unique. Then we say that $\alpha$ is a Brjuno number if

$$
\sum_{n} \frac{\log q_{n+1}}{q_{n}}<\infty
$$

\footnotetext{
* The research of the author was supported in part by the National Science Foundation through grant DMS-9803164 and also by the VIGRE program under National Science Foundation grant DMS-9983646. The author would also like to thank Robert L. Devaney for his invaluable suggestions.
} 
For more detail on the continued fraction expansion, see Section 3.

The Brjuno numbers have previously arisen in the context of "small divisor" problems. One question in complex dynamical systems is the following: Let $f$ be a holomorphic function with a fixed point at the origin, so that $f(0)=0$ and $f^{\prime}(0)=\lambda$. When is $f$ conjugate to its linear part, i.e. when can we find an biholomorphism $h$ so that

$$
h^{-1} \circ f \circ h=f_{L}(z)
$$

with $f_{L}(z)=\lambda z$ ? If we can find such an $h$, we say that $f$ is linearizable.

It was known classically that if $|\lambda| \neq 1$, then one can always find an $h$. The case where $|\lambda|=1$, but $\lambda$ is a root of unity, was also understood (see [11]), although in this case, the answer is sometimes negative.

The difficult case is then when $\lambda=e^{2 \pi i \alpha}$ with $\alpha$ irrational. The reason that this is a "small divisor" problem is that one can always formally find the power series expansion of $h=\sum h_{k} z^{k}$ by the above functional equation, and when one writes down a formula for $h_{k}$, one finds that there is a factor of $\lambda^{k-1}-1$ in the denominator. Since the powers of $\lambda$ form a dense subset of the unit circle, there will be $k$ such that $\lambda^{k-1}-1$ is arbitrarily close to 0 , which will make the $h_{k}$ large. Clearly, if this is true for "many" $h_{k}$, then the power series will not converge. Thus, the convergence of the power series for $h$ depends upon how often the number $\lambda^{k}$ is close to 1 as it winds around the unit circle. If $\alpha$ were rational (and thus $\lambda$ a root of unity), then $\lambda^{k}$ would be exactly 1 for infinitely many $k$. Since $\alpha$ is irrational, the $\lambda^{k}$ will wind around the unit circle densely and never be exactly equal to 1 , but the better one can approximate $\alpha$ with rational numbers, the more often the $\lambda^{k}$ 's will visit a small neighborhood of 1 , making more $h_{k}$ 's large. Thus it is perhaps not surprising that the convergence of the power series of $h$ depends on the continued fraction expansion of $\alpha$.

In 1942, Siegel [11] showed that $f$ is linearizable if $\alpha$ satisfies a Diophantine condition of the form

$$
\left|\alpha-\frac{p}{q}\right|>\frac{\gamma}{q^{\delta}}, \quad \text { for all } q,
$$

for some positive $\gamma$ and $\delta$. Siegel's result is the first time that a small divisor problem was solved [14]. It was also then known that sometimes one could not linearize such an $f$, i.e. that for some irrational $\alpha$, there are functions $f(z)=e^{2 \pi i \alpha} z+\mathcal{O}\left(z^{2}\right)$ which are not linearizable.

Brjuno improved Siegel's result (see [4]) and showed that as long as $\alpha$ is a Brjuno number, then all $f$ 's with $f^{\prime}(0)=e^{2 \pi i \alpha}$ are linearizable. It was not known at the time whether this set of Brjuno numbers was the optimal solution to the problem. Yoccoz, in [14], completely solved the problem, and showed the Brjuno condition is sharp, i.e. if $\alpha$ is not a Brjuno number, 
there there is at least one $f(z)$ with $f^{\prime}(0)=e^{2 \pi i \alpha}$ which is not linearizable. For more details on this linearization question and its history, see [9],[13], $[12],[10]$.

In [5], we studied the symbolic dynamics of the complex exponential family $E_{\lambda}(z)=\lambda e^{z}$. Since this map takes horizontal strips of height $2 \pi$ and maps them over the plane, it is natural to think of these strips as "fundamental domains" of the map. Given a $z \in \mathbb{C}$, we call the itinerary of $z$ to be the integer sequence given by the sequence of strips the point visits.

It was first shown in [7] that if one chooses $\lambda$ so that $E_{\lambda}$ has an attracting fixed point, then the Julia set of $E_{\lambda}$ is a disjoint union of "hairs", sets homeomorphic to the closed half-line. Each hair is invariant, and is made up of the points with a given itinerary. The hairs are indexed by the itineraries, so that the set of all points in the Julia set with a given itinerary lie on one hair. See also [1] for more details of this case. For the case where $\lambda$ is chosen so that $E_{\lambda}$ has an attracting cycle, the hairs are no longer disjoint, but can intersect in a more complicated manner. This has been studied in [6], [2], and [3], although there are still some interesting open questions for even this case. In any case, the symbolic dynamics of the complex exponential are quite useful in understanding the structure of, and the induced dynamics on, the Julia set of $E_{\lambda}$.

For any $\lambda$, we define the set of allowable itineraries as those integer sequences which are obtained as the itinerary of a point under application of the map $E_{\lambda}$. We showed in [5] that this set of allowable itineraries is independent of $\lambda$. Furthermore, it was shown that the itineraries for the map $e^{p(z)}$, where $p$ is any nonconstant polynomial, is independent of $p$. Thus there is some set of integer sequences which is universal for the complex exponential map.

We can think of a sequence of integers as the coefficients of the continued fraction expansion of an irrational number, and can thus consider this set of allowable itineraries as a subset of the irrational numbers. It turns out that this set is related to the Brjuno numbers in a nice way. What we will show is that if one takes the sequences which correspond to Brjuno numbers, and append every sequence which is majorized by the Brjuno sequences (in a sense to be made precise below) then one obtains the allowable itineraries of the complex exponential.

Another connection between complex dynamical systems and the Brjuno numbers which we would like to mention is [9]. Here, the Brjuno numbers are embedded into the plane in a natural way, and the topology of this embedded set is characterized.

In Section 2 we will define more precisely this set of allowable itineraries. In Section 3 we will define precisely the continued fraction expansion and discuss some of its properties. In Section 4 we will define the Brjuno num- 
bers and describe the asymptotic behavior of the continued fraction expansions of Brjuno numbers. Finally, in Section 5 we will prove the main result of the paper.

\section{THE ALLOWABLE ITINERARIES FOR THE COMPLEX EXPONENTIAL}

Let us denote $E(x)=\exp (x)$. We define a set of integer sequences $A$, such that $\mathbf{s}=\left\{s_{j}\right\} \in A$ if there is a real number $x$

$$
\left|s_{j}\right| \leq E^{j}(x),
$$

where $E^{j}$ means the map $E$ iterated $j$ times.

We colloquially say that $A$ contains those sequences which grow no more quickly than an iterated exponential. One can define, more generally, those sequences which do not grow more quickly than the iterates of $e^{\lambda x}$ for $\lambda>0$. It was shown in [5] that this set is independent of $\lambda$. For example, any bounded sequence is in $A$, and, in fact, any unbounded sequence which does not grow too quickly lies in $A$.

We also denote $E_{p}(x)=e^{p(x)}$, where $p$ is a polynomial in $x$. We define the set of itineraries of order $p(x)$ to be the set of sequences

$$
A_{p}=\left\{\mathbf{s} \mid \text { there is an } x>0 \text { such that }\left|s_{j}\right| \leq E_{p}^{j}(x)\right\} .
$$

One of the results of [5] which has already been quoted is that $A_{\lambda x}$ is independent of $\lambda$. In fact, more was shown there; in Section 5 of that paper it is shown that $A_{p(x)}$ is independent of the polynomial $p$.

The main part of the argument was to show that $A_{x^{n}} \subset A_{x}$ (the converse inclusion is clear). To show this, it is sufficient to show that for any $x$, there is a $y$ such that

$$
E_{x^{n}}^{j}(x) \leq E^{j}(y)
$$

By taking $j$ logarithms on each side, one sees that this condition is equivalent to

$$
1+\log n+n x^{n} \leq y
$$

and for fixed $n$ and $x$, one can choose a $y$ to satisfy this. Thus any sequence which grows more slowly than an iterated exponentiated polynomial will be in $A$. On the other hand, not all integer sequences are in $A$. For an example, consider the sequence with

$$
a_{j}=\left\lfloor E^{2 j}(1)\right\rfloor .
$$


where $\lfloor x\rfloor$ denotes the greatest integer contained in $x$. If we assume that there is a fixed $x^{*}$ with

$$
\left|a_{j}\right| \leq E^{j}\left(x^{*}\right)
$$

or

$$
\left\lfloor E^{2 j}(1)\right\rfloor \leq E^{j}\left(x^{*}\right),
$$

then

$$
\begin{aligned}
E^{2 j}(1) & \leq E^{j}\left(x^{*}\right)+1 \leq E^{j}\left(x^{*}+1\right) \\
E^{j}(1) & \leq x^{*}+1
\end{aligned}
$$

which is a contradiction.

\section{THE CONTINUED FRACTION EXPANSION}

The classical continued fraction expansion, which we will describe below, takes an irrational number and gives a sequence of positive integers. Our set $A$ has both positive and negative entries. There is a continued fraction algorithm (see for example [13]) which allows for negative entries. However, in the interests of simplicity and familiarity, we want to use the classical expansion. So we are going to consider the set of all positive integer sequences $\left\{t_{n}\right\}$ that have the property that there is an $x^{*}$ with $t_{j} \leq E^{j}\left(x^{*}\right)$. Let us call this set $\tilde{A}$.

We should note that we can get $\tilde{A}$ simply by relabelling the elements of $A$. Define the map on integer sequences by $n \rightarrow 2 n$ if $n$ is positive and $n \rightarrow-2 n+1$ if $n$ is negative. Then if $\mathbf{s} \in A$, we have

$$
\left|s_{j}\right| \leq E^{j}\left(x^{*}\right) .
$$

But then $t_{j} \leq 2\left|s_{j}\right|+1$, so that we have

$$
t_{j} \leq E^{j}\left(2 x^{*}+1\right) .
$$

We will actually abuse notation by also calling this new set $A$. So, in short, we are now considering the set $A$ of positive integer sequences which grow no more quickly than an iterated exponential.

Consider the map $\phi$ from infinite positive integer sequences to irrational numbers as follows:

$$
\phi\left(\left\{a_{0}, a_{1}, \ldots\right\}\right)=a_{0}+\frac{1}{a_{1}+\frac{1}{a_{2}+\frac{1}{a_{3}+\ddots}}}
$$


It is a classical fact of number theory [8] that this map is one-to-one and onto from the set of infinite integer sequences to the irrationals. (An analogous map exists from the set of finite integer sequences to the rationals, but it is two-to-one.)

The question we are concerned with in this paper is: What is $\phi(A)$, and how does it relate to the Brjuno numbers?

\section{THE BRJUNO NUMBERS}

Consider an infinite integer sequence $\left\{a_{0}, a_{1}, \ldots\right\}, \alpha=\phi\left(\left\{a_{0}, a_{1}, \ldots\right\}\right)$. Given a finite subsequence $\left\{a_{0}, a_{1}, \ldots, a_{n}\right\}$, let us denote

$$
\left\langle a_{0}, a_{1}, \ldots, a_{n}\right\rangle=a_{0}+\frac{1}{a_{1}+\frac{1}{a_{2}+\frac{1}{a_{3}+\ddots}+\frac{1}{a_{n}}}},
$$

which is a rational number, as $p_{n} / q_{n}$.

The number $p_{n} / q_{n}$ is known as the $n$-th convergent of $\alpha$. Classical theory (again see [8]) has much to say about these numbers. For example, $p_{n} / q_{n} \rightarrow$ $\alpha$, and, moreover, this is the sequence of rationals which converges to $\alpha$ most quickly, by which we mean that $p_{n} / q_{n}$ is closer to $\alpha$ than any other rational number with the same or smaller denominator. It can also be calculated that

$$
\begin{array}{lll}
p_{0}=a_{0} & p_{1}=a_{0} a_{1}+1 & p_{n}=a_{n} p_{n-1}+p_{n-2} \\
q_{0}=1 & q_{1}=a_{1} & q_{n}=a_{n} q_{n-1}+q_{n-2} .
\end{array}
$$

It should be noted from this recursive formula that, given an infinite integer sequence $\left\{a_{n}\right\}$, the two sequences $\left\{p_{n}\right\}$ and $\left\{q_{n}\right\}$ can be generated without referring to the continued fraction expansion at all.

Definition 1. The Brjuno sum of a number $\alpha \notin \mathbb{Q}$ is

$$
B(\alpha):=\sum_{n} \frac{\log q_{n+1}}{q_{n}},
$$

and the Brjuno numbers are the irrational numbers for which this sum is finite. We denote the set of Brjuno numbers by $B$. We will abuse notation by saying that an infinite integer sequence $\left\{a_{n}\right\}$ is Brjuno if $\phi\left(\left\{a_{n}\right\}\right)$ is a Brjuno number. 
The main theorem of this section is

THEOREM 2. Let $\alpha$ be irrational. If the corresponding $a_{n}$ satisfy the inequality

$$
a_{n+1}<a_{n}^{\rho a_{n}}, \quad \text { with some } \rho<1,
$$

except for finitely many $n$, then $\alpha \in B$. If the corresponding $a_{n}$ satisfy

$$
a_{n+1}>a_{n}^{\sigma a_{n}}, \quad \text { with some } \sigma>1,
$$

except for finitely many $n$, then $\alpha \notin B$.

We note that this theorem could be strengthened; as one will see in the proof, the statement could be much sharper. For the purposes of this paper, this theorem is sufficient. To prove this theorem, we need a few lemmas:

LEMMA 3.

$$
\log \left(a_{n}+\frac{1}{a_{n-1}}\right)<\log \left(a_{n}\right)+1
$$

Proof. Observe that $\log (x+1)<\log (x)+1$, if $x>1 /(e-1)$.

Lemma 4. For any choice of the sequence $\left\{a_{n}\right\}$,

$$
q_{n} \geq(\sqrt{2})^{(n-1)},
$$

with the inequality strict for $n \geq 2$.

Proof. We will show the theorem for the choice of all $a_{n}=1$. This clearly suffices, since if we increase any of the $a_{n}$, all subsequent $q_{n}$ also increase.

Then we have $q_{0}=1>1 / \sqrt{2}, q_{1}=1, q_{2}=2>\sqrt{2}$. Assume the conclusion is true for $q_{i}$ with $i \leq n$. Then

$$
\begin{aligned}
q_{n+1} & =a_{n+1} q_{n}+q_{n-1}>(\sqrt{2})^{n-1}+(\sqrt{2})^{n-2} \\
& =(\sqrt{2})^{n-2}(\sqrt{2}+1)>(\sqrt{2})^{n} .
\end{aligned}
$$

LEMmA 5. The sum $\sum_{n}\left(\frac{\log q_{n+1}}{q_{n}}\right)$ converges iff $\sum_{n}\left(\frac{\log a_{n+1}}{q_{n}}\right)$ converges. 
Proof. One direction is trivial since $q_{n}>a_{n}$, so we will prove only the other direction. Since $q_{n+1}>a_{n+1} q_{n}$, we have

$$
q_{n+1}=\left(a_{n+1}+\frac{q_{n-1}}{q_{n}}\right) q_{n}<\left(a_{n+1}+\frac{1}{a_{n}}\right) q_{n}
$$

Using Lemma 3, we have that

$$
\begin{aligned}
\sum_{n} \frac{\log \left(q_{n+1}\right)}{q_{n}} & <\sum_{n} \frac{\log \left(a_{n+1}+\frac{1}{a_{n}}\right)+\log \left(q_{n}\right)}{q_{n}} \\
& <\sum_{n}\left(\frac{\log \left(a_{n+1}\right)}{q_{n}}+\frac{1}{q_{n}}+\frac{\log \left(q_{n}\right)}{q_{n}}\right)
\end{aligned}
$$

Since everything is positive, we have that

$$
\sum_{n}\left(\frac{\log \left(a_{n+1}\right)}{q_{n}}+\frac{1}{q_{n}}+\frac{\log \left(q_{n}\right)}{q_{n}}\right)=\sum_{n} \frac{\log \left(a_{n+1}\right)}{q_{n}}+\sum_{n} \frac{1}{q_{n}}+\sum_{n} \frac{\log \left(q_{n}\right)}{q_{n}} .
$$

and using Lemma 4 , and the fact that $\log (x) / x$ is monotone decreasing for $x$ large, we have

$$
\sum_{n} \frac{1}{q_{n}}<\sum_{n} \frac{\log \left(q_{n}\right)}{q_{n}}<\sum_{n} \frac{(n-1) \log \sqrt{2}}{(\sqrt{2})^{(n-1)}}<\infty .
$$

Remark 6. We have shown that a number $\alpha \in B$ if and only if the sum

$$
\sum_{n} \frac{\log a_{n+1}}{q_{n}}<\infty
$$

so this could be the definition. It turns out that while this is more convenient for our purposes, the classical definition is more natural and makes more sense from the perspective of the linearization question.

Proof (Proof of Theorem 2). First, let us assume that $a_{n+1}<a_{n}^{\rho a_{n}}$, for $\rho<1$, except for finitely many $n$. We will check Equation 3 using the Ratio Test. As long as our hypothesis holds,

$$
\begin{aligned}
\frac{\log \left(a_{n+1}\right)}{q_{n}} \frac{q_{n-1}}{\log \left(a_{n}\right)} & <\frac{\rho a_{n} \log \left(a_{n}\right) q_{n-1}}{q_{n} \log \left(a_{n}\right)} \\
& =\rho a_{n} \frac{q_{n-1}}{q_{n}}<\rho<1
\end{aligned}
$$


Since our hypothesis (and thus Equation 4) holds in all but a finite number of cases, it holds in the limit.

Now, assume that $a_{n+1}>a_{n}^{\sigma a_{n}}$, with $\sigma>1$. Then using the Ratio Test, we have

$$
\begin{aligned}
\frac{\log a_{n+1}}{q_{n}} \frac{q_{n-1}}{\log a_{n}} & >\frac{\sigma a_{n} \log \left(a_{n}\right) q_{n-1}}{\log \left(a_{n}\right) q_{n}} \\
& >\sigma a_{n} \frac{q_{n-1}}{q_{n}}=\sigma\left(1-\frac{q_{n-2}}{q_{n}}\right) .
\end{aligned}
$$

Clearly $q_{n}>a_{n} a_{n-1} q_{n-2}$. Since the $a_{n}$ are growing quickly, the last line of Equation 5) gets as close to $\sigma$ as needed, so is larger than 1 in the limit.

\section{THE CHARACTERIZATION OF $\phi(A)$}

We want to characterize $\phi(A)$ as a set of irrational numbers. In view of Theorem 2, a first guess would be that the set $\phi(A)$ is equal to the Brjuno numbers, since both of these sets have the property that they grow (almost) like an iterated exponential map. We will compare $A$ and $\phi^{-1}(B)$ below. We will abuse terminology by referring to an integer sequence being Brjuno if the associated irrational number is, and also denoting $\phi^{-1}(B)$ as $B$, and the context will make it clear which we mean. In short, $B$ can mean the Brjuno numbers or the set of sequences which give rise to the Brjuno numbers.

Let us put a partial ordering on integer sequences as follows: If $\mathbf{a}=\left\{a_{n}\right\}$ and $\mathbf{b}=\left\{b_{n}\right\}$, then we say $\mathbf{a} \preceq \mathbf{b}$ if

$$
\left|a_{n}\right| \leq\left|b_{n}\right| \text { for all } n \text {. }
$$

Definition 7. We say a set $C$ of infinite integer sequences is full if, whenever $\mathbf{a} \in C$, then $\mathbf{b} \in C$ for all $\mathbf{b} \preceq \mathbf{a}$.

THEOREM 8. B is not full.

Proof. We denote $\lceil x\rceil$ to be the smallest integer greater than or equal to $x$. From Theorem 2, we know that if we have a sequence $\left\{a_{n}\right\}$ with the recursive relation

$$
a_{n+1}=\left\lceil e^{a_{n}^{2}}\right\rceil
$$

then $\left\{a_{n}\right\} \notin B$. Since $\left\{a_{n}\right\} \in A_{x^{2}}=A_{x / 2}$, so that there is an $x^{*}$ such that

$$
\left|a_{n}\right| \leq E_{x / 2}^{n}\left(x^{*}\right)
$$


Assume without loss of generality that $x^{*}>1$, and define $\mathbf{b}=\left\{b_{n}\right\}$ by

$$
b_{n}=\left\lfloor E_{x / 2}^{n}\left(x^{*}+1\right)\right\rfloor .
$$

It is easy to see that $\left(E_{x / 2}^{n}\left(x^{*}+1\right)-E_{x / 2}^{n}\left(x^{*}\right)\right) \geq 1$, and thus $b_{n} \geq a_{n}$ for any $n$. From the definition of $b_{n}, b_{n+1} \leq \exp \left(b_{n} / 2\right)$, so that by Theorem 2 , the sequence $\left\{b_{n}\right\}$ is a Brjuno sequence.

In summary, we have $\mathbf{a} \preceq \mathbf{b}$, but $\mathbf{a} \notin B$ and $\mathbf{b} \in B$.

Since it is obvious by definition that $A$ is full, we see that $A \neq B$. But the next best thing is true: $A$ is equal to the smallest full set of sequences which contains $B$, which we call $B^{\text {full }}$.

TheOREM 9. $B^{\text {full }}=A$.

Proof. $\supseteq$ : Let us assume that $\left\{a_{n}\right\} \in A$. Then there is an $x^{*}>1$ with

$$
\left|a_{n}\right| \leq E_{x / 2}^{n}\left(x^{*}\right) .
$$

Define $\alpha_{n}=\left\lceil E_{x / 2}^{n}\left(x^{*}\right)\right\rceil$. Clearly, $\left\{a_{n}\right\} \preceq\left\{\alpha_{n}\right\}$. Also

$$
\alpha_{n+1} \leq\left\lceil e^{\alpha_{n} / 2}\right\rceil \leq e^{\alpha_{n} / 2}+1<\alpha_{n}^{\alpha_{n} / 2}
$$

for $\alpha_{n}>2 e^{2}$. This is certainly true for $n$ large enough, and then

$$
\alpha_{n+1} \leq \alpha_{n}^{\alpha_{n} / 2}
$$

for all but finitely many $n$, and thus $\left\{\alpha_{n}\right\} \in B$. Since $\left\{a_{n}\right\} \preceq\left\{\alpha_{n}\right\}$, we have $\left\{a_{n}\right\} \in B^{\text {full }}$.

$\subseteq$ : We assume that $\left\{a_{n}\right\} \in B^{\text {full }}$, or that there is an integer sequence $\left\{b_{n}\right\} \succeq\left\{a_{n}\right\}$ with $\left\{b_{n}\right\} \in B$. This means that (see Theorem 2) that

$$
b_{n+1} \leq b_{n}^{2 b_{n}}
$$

for all but a finite number of $n$. Stated another way, there is an $N$ such that for all $n \geq N$, Equation 6 is true. Define $c_{n}=b_{N+n}$. First, we show that $\left\{c_{n}\right\} \in A$. It is clear that for all $n$,

$$
c_{n+1} \leq c_{n}^{2 c_{n}}=E_{2 x}\left(c_{n}\right),
$$

so that $\left\{c_{n}\right\} \in A_{2 x}=A$. There is an $x^{*}>1$ such that

$$
\left|c_{n}\right| \leq E^{n}\left(x^{*}\right)
$$

but then

$$
\left|b_{n+N}\right|=\left|c_{n}\right| \leq E^{n}\left(x^{*}\right)<E^{n+N}\left(x^{*}\right),
$$


so that $\left\{b_{n}\right\} \in A$. Then, since $A$ is full and $\mathbf{a} \preceq \mathbf{b}$, we have that $\left\{a_{n}\right\} \in$ A.

We can think of $A$ as being a sort of star-shaped hull of $B$, in the sense that $A$ is the smallest star-shaped set which contains $B$. This is because we can connect any element in $B$ to the origin by decreasing entries, and all of these intermediate sequences are elements of $A$.

\section{CONCLUSIONS}

We have described the connection between the Brjuno numbers and the itineraries of the complex exponential. One question which can be posed is whether this connection has any implications for the study of the dynamics of the complex exponential map, and unfortunately, we do not see any at this time. Nonetheless, this is another interesting connection between the dynamics of complex analytic maps and number theory.

\section{REFERENCES}

1. J.M. Aarts and L.G. Oversteegen, The geometry of Julia sets, Trans. Amer. Math. Soc., 338 (1993), Number 2, 897-918.

2. R. Bhattacharjee And R.L. Devaney, Tying hairs for structurally stable exponentials, Ergodic Theory Dynam. Systems, 20 (2000), Number 6, 1603-1617.

3. , R. Bhattacharjee, R.L. Devaney, R.E. Lee Deville, K. Josić and M. MorenoRocha, Accessible points in the Julia sets of stable exponentials, Discrete Contin. Dyn. Syst. Ser. B, 1 (2001), Number 3, 299-318.

4. A.D. BRJuno, On convergence of transforms of differential equations to the normal form, Dokl. Akad. Nauk SSSR, 165 (1965), 987-989.

5. R.E. LeE DeVille, Itineraries of entire functions, J. Differ. Equations Appl., 7 (2001), Number 2, 193-214.

6. R.L. Devaney and X. Jarque, Misiurewicz points for complex exponentials, Internat. J. Bifur. Chaos Appl. Sci. Engrg., 7 (1997), Number 7, 1599-1615.

7. R.L. Devaney and M. Krych, Dynamics of $\exp (z)$, Ergodic Theory Dynam. Systems, 4 (1984), Number 1, 35-52.

8. G. H. Hardy and E. M. Wright, An introduction to the theory of numbers, The Clarendon Press Oxford University Press, New York, fifth edition (1979).

9. E.F. LeE, The structure and topology of the Brjuno numbers, Proceedings of the 1999 Topology and Dynamics Conference (Salt Lake City, UT), 24 (1999), 189-201.

10. R. PÉrez-MARCo, Sur les dynamiques holomorphes non linéarisables et une conjecture de V. I. Arnol'd, Ann. Sci. École Norm. Sup. (4), 26 (1993), Number 5, $565-644$.

11. C.L. Siegel, Iteration of analytic functions, Ann. of Math., 43 (1942), Number 2, 607-612.

12. C.L. Siegel And J.K. Moser, Lectures on celestial mechanics, Springer-Verlag, New York (1971). Translation by Charles I. Kalme, Die Grundlehren der mathematischen Wissenschaften, Band 187. 
13. J.C. YocCOz, Conjugaison différentiable des difféomorphismes du cercle dont le nombre de rotation vérifie une condition diophantienne, Ann. Sci. École Norm. Sup. (4), 17 (1984), Number 3, 333-359.

14. J.C. YocCOZ, Théorème de Siegel, nombres de Bruno et polynômes quadratiques, Astérisque, 231 (1995), 3-88. Petits diviseurs en dimension 1. 